\title{
$\Delta$ Np63 drives metastasis in breast cancer cells via PI3K/CD44v6 axis
}

\author{
Simone Di Franco ${ }^{1, *}$, Alice Turdo ${ }^{1, *}$, Antonina Benfante ${ }^{1}$, Maria L. Colorito ${ }^{1,2}$, \\ Miriam Gaggianesi ${ }^{1}$, Tiziana Apuzzo ${ }^{1}$, Raju Kandimalla ${ }^{3}$, Aurora Chinnici ${ }^{1}$, Daniela \\ Barcaroli $^{4}$, Laura Rosa Mangiapane ${ }^{1}$, Giuseppe Pistone ${ }^{5}$, Salvatore Vieni ${ }^{1}$, Eliana \\ Gulotta $^{1}$, Francesco Dieli ${ }^{6}$, Jan Paul Medema ${ }^{3}$, Giorgio Stassi ${ }^{1}$, Vincenzo De \\ Laurenzi ${ }^{2}$ and Matilde Todaro ${ }^{6,7}$ \\ ${ }^{1}$ Department of Surgical and Oncological Sciences, Cellular and Molecular Pathophysiology Laboratory, University of Palermo, \\ Palermo, Italy \\ 2 Dipartimento di Scienze Mediche, Orali e Biotecnologiche, University "G. d'Annunzio" Chieti-Pescara, CESI-MeT, Chieti, Italy \\ 3 Laboratory for Experimental Oncology and Radiology, Center for Experimental Molecular Medicine, Academic Medical \\ Center, University of Amsterdam, Amsterdam, The Netherlands \\ ${ }^{4}$ DISPUTer, University "G. d'Annunzio" Chieti-Pescara, Chieti, Italy \\ ${ }^{5}$ Department of DIBIMIS, Dermatology Section, University of Palermo, Palermo, Italy \\ ${ }^{6}$ Central Laboratory of Advanced Diagnosis and Biomedical Research (CLADIBIOR), University of Palermo, Palermo, Italy \\ 7 DiBiMIS, University of Palermo, Palermo, Italy \\ * These authors have contributed equally to this work \\ Correspondence to: Matilde Todaro, email: matilde.todaro@unipa.it
}

Vincenzo De Laurenzi, email: delaurenzi@unich.it

Keywords: breast cancer initiating cells, p63, PI3K/AKT pathway, CD44v6, metastasis

Received: June 20, $2016 \quad$ Accepted: July 22, $2016 \quad$ Published: August 02, 2016

\section{ABSTRACT}

P63 is a transcription factor belonging to the family of p53, essential for the development and differentiation of epithelia. In recent years, it has become clear that altered expression of the different isoforms of this gene can play an important role in carcinogenesis. The $p 63$ gene encodes for two main isoforms known as TA and $\Delta \mathbf{N}$ p63 with different functions. The role of these different isoforms in sustaining tumor progression and metastatic spreading however has not entirely been clarified.

Here we show that breast cancer initiating cells express $\Delta N p 63$ isoform that supports a more mesenchymal phenotype associated with a higher tumorigenic and metastatic potential. On the contrary, the majority of cells within the tumor appears to express predominantly TAp63 isoform. While $\Delta N p 63$ exerts its effects by regulating a PI3K/CD44v6 pathway, TAp63 modulates this pathway in an opposite fashion. As a result, tumorigenicity and invasive capacity of breast cancer cells is a balance of the two isoforms. Finally, we found that tumor microenvironmental cytokines significantly contribute to the establishment of breast cancer cell phenotype by positively regulating $\Delta \mathrm{Np} 63$ and CD44v6 expression.

\section{INTRODUCTION}

The development of epithelial tissue is a finely regulated process that involves several transcriptional factors [1]. p63, a p53 homolog, plays a key role in the generation of all the stratified squamous epithelia and their derivatives, including breast $[2,3]$.
The $p 63$ gene encodes six different isoforms, based on the presence of an amino-terminal trans-activation domain (TAp63) or its absence ( $\triangle N p 63)$, which can undergo alternative splicing at the 3 ' end of the gene to generate the $\alpha, \beta$ and $\gamma$ isoforms $[4,5]$. Their structure suggests that TAp63 and $\Delta \mathrm{Np} 63$ have distinct and opposing functions. 
The role of p63 in stratified epithelium development was determined using murine genetic models. p63 KO mice showed alterations of all stratified epithelia as well as of epithelial appendages, including the mammary gland. The interpretation of these results about the role of p63 in epithelial development is still controversial and different models have been proposed. One model suggests that p63 is essential for the commitment and differentiation of epithelial precursors, while the other proposes that it is essential for the maintenance of the progenitor population $[2,3]$. A third model attempts to reconcile the previous ones, suggesting that $\Delta \mathrm{Np} 63$ is essential for the maintenance of the progenitor population while TAp63 is necessary to allow complete differentiation [6-9].

p63 has a major role in breast development, in the mature mammary gland it is confined to the early mammary progenitors and myoepithelial/basal cells, where $\Delta \mathrm{Np} 63$ is the predominant isoform $[10,11] . \Delta \mathrm{Np} 63$ enhances cell proliferation [12] and inhibits apoptosis [13], whereas TAp63 induces apoptosis [14] and inhibits cell-cycle progression [15] leading to cell differentiation.

Current models describe healthy and cancer tissue growth as driven by stem cells. In this context, genetic/ epigenetic changes that affect normal stem cells can lead to production and expansion of cancer initiating cells (CICs) [16, 17], which have been isolated and characterized in many solid tumors, including breast $[18,19]$. Recent research has shown that the origin of normal and cancer stem cells in breast tissue may also be caused by de-differentiation of committed healthy and cancer cells, thus making the cellular heterogeneity and hierarchy more complex [20]. This plasticity seems to be regulated by the microenvironment and mainly through the induction of an EMT programme. It was also recently demonstrated that breast CICs (BCICs) exist in distinct mesenchymal- and epithelial-like states that are characterized by specific markers and gene-expression profiles [21]. Interestingly, the mesenchymal-like BCICs have a similar gene-expression profile to basal stem cells, whereas the epithelial-like BCICs show luminal stem cell traits that are found in normal breast tissue.

CD44v6 has recently been described as both a functional biomarker and a therapeutic target of metastatic $\mathrm{CICs}$, through the activation of PI3K signaling [22]. Of note, the PI3K pathway has been demonstrated to play an important function in the development of breast cancer, as it is deregulated in almost $50 \%$ of patients [23]. For these reasons, PI3K pathway is considered an attractive biological target, to be mainly applied in the treatment of the most aggressive tumors [24].

Here we show that breast cancer sphere cells (BCSCs), known to be enriched in cells with cancer initiating/stem-like features [16], are characterized by the expression of $\Delta \mathrm{Np} 63$, which is crucial for the induction of an EMT programme, thus increasing the ability of breast cancer cells to migrate and form distant metastasis, through the activation of PI3K/AKT pathway and CD44v6 expression. Differently, cells bearing the TAp63 expression are endowed with tumorigenic potential but unable to form metastasis and tumors after serial transplantation, showing reduced levels of $\mathrm{PI} 3 \mathrm{~K} / \mathrm{AKT}$ pathway activation. The selective targeting of CD44v6 led to a decrease of BCSCs proliferation, clonogenicity and invasion in vitro. Finally, tumor microenvironmental stimuli played a key role in the promotion of the BCSC phenotype that regulates the expression levels of $\triangle \mathrm{Np} 63$ and CD44v6.

\section{RESULTS}

\section{BCSCs with high tumorigenic and metastatic potential express higher levels of $\Delta \mathrm{Np} 63$}

We freshly isolated breast cancer cells from surgical breast cancer resections. As detailed in the material and methods section, we cultured them either as sphere cells (Suppl. Figure 1A), referred to as BCSCs, or as sphere derived adherent cells (SDACs) (Suppl. Figure 1A). In order to establish the luminal and basal phenotype of our collection of breast cancer samples, we assessed the expression of clinical markers associated with specific breast cancer molecular subtypes [25-27] in formalinembedded histological samples. In accordance with the well established characterization of breast carcinoma subtypes [18, 25, 26], luminal breast cancer samples were characterized by $\mathrm{CK} 8-18^{+}, \mathrm{CK} 5^{-}, \mathrm{CK} 14^{-}, \mathrm{ER}^{+}$, $\mathrm{PR}^{+/}, \mathrm{HER}^{+/-}, \mathrm{ALDH}^{-}$, while basal breast carcinomas were CK8-18-, $\mathrm{CK}^{+}, \mathrm{CK}_{14}^{+}, \mathrm{ER}^{-}, \mathrm{PR}-\mathrm{HER} 2^{-}, \mathrm{ALDH}^{+}$ [25] (Table 1, Figure 1A). Interestingly and in line with previous findings [28], basal breast cancers and their derived BCSCs showed higher expression of p63, as compared to the luminal samples (Figure 1A). Moreover, basal BCSCs retained higher levels of CK5, CK14, CD10 and VIMENTIN (Suppl. Figure 1B-1C).

Although both luminal and basal BCSCs were capable of forming tumors when subcutaneously injected in NOD/SCID mice, basal BCSCs showed an enhanced tumorigenic potential than luminal ones (Figure 1B). The tumorigenic potential was lost upon deprivation of EGF and b-FGF followed by exposure to serum (SDACs), as shown by failure to form tumors despite the injection of high number $\left(10^{6}\right)$ of SDACs (Figure 1B).

Both basal BCSC primary lines derived from patient \#10 and \#30 retained in vitro invasive capacity and in vivo metastatic ability when injected under the renal capsule of immunodeficient mice (Figure 1C-1D). In contrast, luminal BCSCs were less invasive in vitro and generated only small primary tumors and were deficient for metastatic spread to lung and bone in vivo (Figure 1D).

We then further investigated mRNA expression of the different $p 63$ isoforms in our samples finding that both 

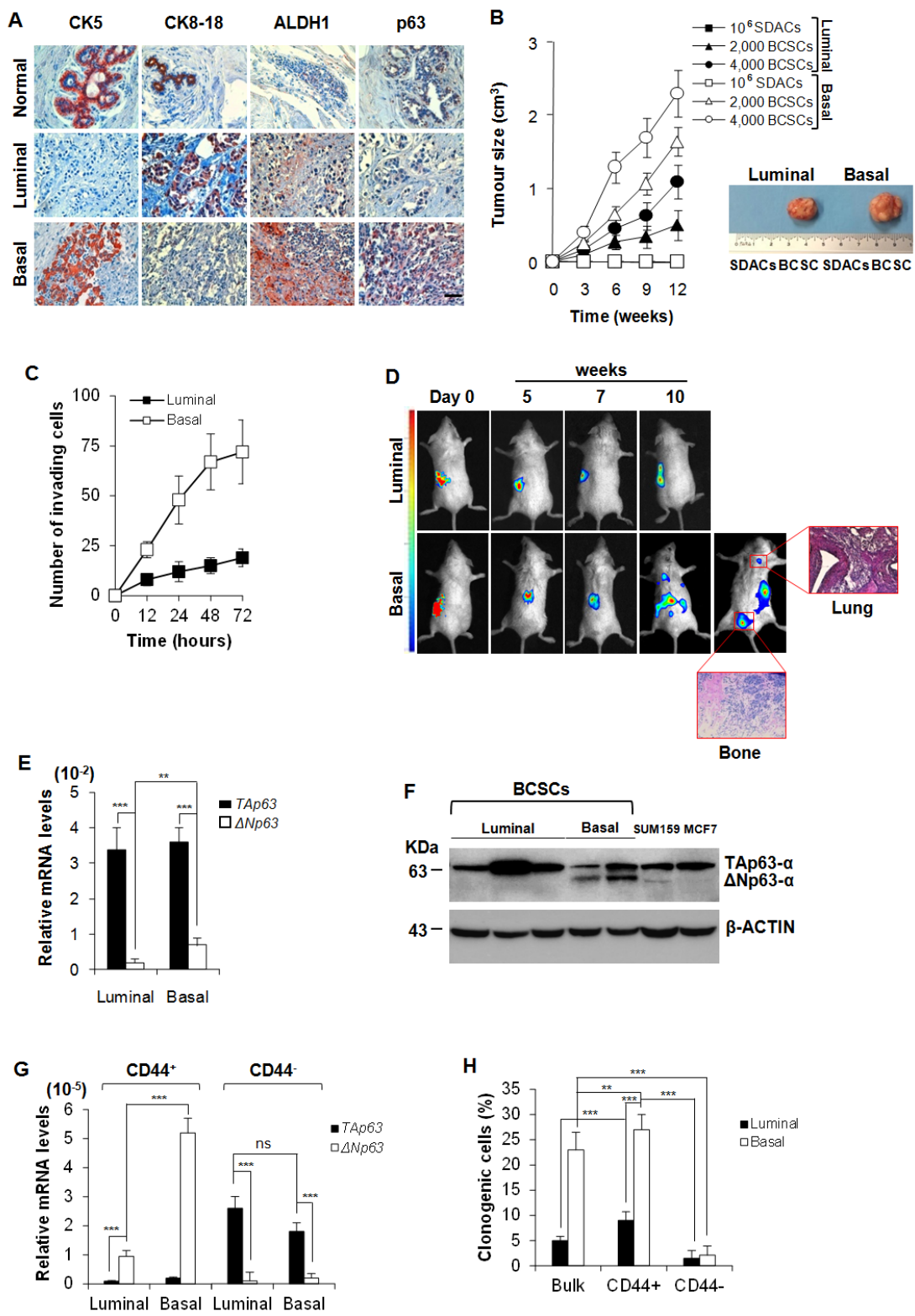

Figure 1: Basal BCSCs express $\triangle \mathrm{Np63}$ and retain a metastatic activity. A. Representative immunohistochemical analysis for CK5, CK8-18, ALDH1 and p63 performed on paraffin embedded sections of 21 breast cancer clinical samples (15 luminal and 6 basal breast cancers) and 6 normal tissues. Scale bar represents $50 \mu \mathrm{m}$. B. Size of subcutaneous tumors generated by the injection of $10^{6} \mathrm{SDACs}$, and 2,000 or 4,000 luminal and basal BCSCs, derived from the same patient. Data are mean \pm SD of 3 independent experiments, performed with cells from 2 luminal and 2 basal different cancer patients. (Right panel) Representative gross morphology of tumors outgrowth derived by implantation of SDACs or 4,000 BCSCs derived from the same patient at 12 weeks. C. Invasion assay of 2,000 luminal and basal BCSCs cultured in SFM up to 72 hours. Results are shown as mean \pm S.D. of 3 independent experiments performed in 3 luminal and 2 basal different primary cell lines. D. In vivo whole-body imaging analysis of sub-renal capsule tumors and metastasis growth at the indicated time points. Xenografts were generated injecting 4,000 BCSCs into the sub-renal capsule. Data are representative of 16 tumor xenografts generated by the injection of 2 luminal and 2 basal BCSCs derived from different patients. (Red boxes) Representative H\&E analysis of lung and bone metastasis generated by injection of basal BCSCs at 10 weeks. E. TAp63 and $\triangle N p 63$ mRNA expression levels in freshly purified luminal and basal breast cancer cells. GAPDH amplification was used as endogenous control. Results show mean \pm S.D. of 3 independent experiments using 5 luminal and 5 basal cancer samples. F. Immunoblot analysis for TAp63- and $\Delta$ Np63- $\alpha$ in luminal and basal BCSCs. SUM159 and MCF7 cell lines were used as basal and luminal control, respectively. Data are representative of 3 independent experiments using 5 BCSC lines derived from 3 luminal and 2 basal breast cancer patients. G. mRNA expression levels of TAp63 and $\triangle N p 63$ in enriched CD44 ${ }^{+}$and CD44- luminal and basal BCSCs. Data are mean \pm S.D. of 3 independent experiments. (H) Clonogenic assay of bulk BCSCs, CD44 ${ }^{+}$and CD44- enriched luminal and basal BCSCs. Data are expressed as mean \pm S.D. of 3 independent experiments performed with BCSCs purified from 3 luminal and 2 basal cancer patients. * indicates $P<0.05$, ** indicate $P<0.01$ and *** indicate $P<$ 0.001. ns indicates non statistically significant. 
Table 1: Breast cancer case description

\begin{tabular}{|c|c|c|c|c|c|c|c|c|c|c|}
\hline Sample & Age & $\begin{array}{l}\text { Tumor } \\
\text { type }\end{array}$ & Grading & ER & PR & \begin{tabular}{|l} 
HER-2/ \\
neu
\end{tabular} & Ki67 & p63 & $\begin{array}{l}\text { Mammosphere } \\
\text { formation }\end{array}$ & Xenograft \\
\hline Pt \#1 & 47 & IDC & $\mathrm{G} 2$ & + & + & - & $25 \%$ & + & No & - \\
\hline Pt \#2 & 65 & IDC & G3 & - & - & ++++ & $>10 \%$ & ++ & $\mathrm{No}$ & - \\
\hline$P t \# 3$ & 63 & IDC & G2 & + & + & - & $20 \%$ & + & $\mathrm{No}$ & - \\
\hline $\mathrm{Pt} \# 4$ & 55 & IDC & G2 & + & + & +++ & $>10 \%$ & + & Yes & $7 / 10$ \\
\hline Pt \#5 & 85 & IDC & G2 & - & - & ++ & $15 \%$ & + & No & - \\
\hline Pt \#6 & 57 & IDC & G2 & + & - & + & $20 \%$ & + & No & - \\
\hline Pt \#7 & 46 & IDC & G3 & + & + & ++ & $15 \%$ & + & Yes & $4 / 10$ \\
\hline$P t \# 8$ & 68 & IDC & G3 & + & + & + & $20 \%$ & + & No & - \\
\hline Pt \#9 & 86 & IDC & G2 & + & + & ++ & $15 \%$ & + & No & - \\
\hline Pt \#10 & 85 & ILC & G2 & - & - & + & $>10 \%$ & ++ & Yes & $10 / 10$ \\
\hline Pt \#11 & 70 & IDC & G2 & + & + & - & $10 \%$ & + & $\mathrm{No}$ & - \\
\hline Pt \#12 & 59 & ILC & G2 & + & + & - & $<10 \%$ & + & $\mathrm{No}$ & - \\
\hline Pt \#13 & 69 & IDC & G3 & + & + & ++ & $10 \%$ & + & $\mathrm{No}$ & - \\
\hline Pt \#14 & 53 & IDC & G2 & + & + & - & $5 \%$ & + & Yes & ND \\
\hline Pt \#15 & 83 & IDC & G3 & - & + & +++ & 10 & ++ & Yes & - \\
\hline Pt \#16 & 67 & IDC & G3 & + & + & - & $10 \%$ & - & No & - \\
\hline Pt \#17 & 56 & IDC & G2 & + & + & ++ & $10 \%$ & + & No & - \\
\hline Pt \#18 & 74 & IDC & G2 & + & + & +++ & $>10 \%$ & + & Yes & $6 / 10$ \\
\hline Pt \#19 & 52 & IDC & G3 & + & + & - & $10 \%$ & + & No & - \\
\hline Pt \#20 & 53 & IDC & G2 & + & + & + & $10 \%$ & + & No & - \\
\hline Pt \#21 & 71 & IDC & G3 & + & + & ++ & $25 \%$ & ++ & No & - \\
\hline Pt \#22 & 69 & IDC & G2 & + & + & + & $25 \%$ & + & Yes & $3 / 10$ \\
\hline Pt \#23 & 85 & IDC & G2 & + & + & - & $15 \%$ & + & $\mathrm{No}$ & - \\
\hline Pt \#24 & 69 & IDC & G2 & + & + & - & $>10 \%$ & + & Yes & - \\
\hline Pt \#25 & 77 & IDC & G2 & + & + & - & $20 \%$ & + & No & - \\
\hline Pt \#26 & 64 & IDC & G2 & + & + & ++ & $25 \%$ & + & No & - \\
\hline Pt \#27 & 73 & IDC & G3 & + & + & + & $20 \%$ & + & No & - \\
\hline $\mathrm{Pt} \# 28$ & 80 & IDC & G3 & + & + & - & $15 \%$ & + & No & - \\
\hline Pt \#29 & 71 & IDC & G3 & + & + & + & $2 \%$ & + & No & - \\
\hline Pt \#30 & 52 & IDC & G3 & - & - & - & $>10 \%$ & $\begin{array}{ll}+++ \\
\end{array}$ & Yes & $10 / 10$ \\
\hline Pt \#31 & 81 & IDC & G2 & + & + & ++ & $30 \%$ & ++ & No & - \\
\hline Pt \#32 & 67 & IDC & G2 & + & + & - & $10 \%$ & + & No & - \\
\hline Pt \#33 & 63 & IDC & G2 & + & + & - & $<10 \%$ & + & No & - \\
\hline Pt \#34 & 75 & IDC & G2 & + & + & - & $35 \%$ & ++ & $\mathrm{No}$ & - \\
\hline Pt \#35 & 86 & IDC & G3 & + & - & ++ & $15 \%$ & + & No & - \\
\hline Pt \#36 & 48 & IDC & G3 & + & + & +++ & $35 \%$ & + & $\mathrm{No}$ & - \\
\hline Pt \#37 & 68 & IDC & G3 & + & + & ++ & $15 \%$ & + & No & - \\
\hline Pt \#38 & 76 & IDC & G3 & + & + & ++ & $30 \%$ & + & No & - \\
\hline Pt \#39 & 65 & IDC & G3 & - & - & +++ & $80 \%$ & ++ & Yes & ND \\
\hline $\mathrm{Pt} \# 40$ & 53 & IDC & G2 & + & + & - & $5 \%$ & + & Yes & ND \\
\hline $\mathrm{Pt} \# 41$ & 76 & IDC & G2 & + & + & - & $10 \%$ & + & No & - \\
\hline $\mathrm{Pt} \mathrm{\# 42}$ & 61 & IDC & G2 & + & + & - & $45 \%$ & + & No & - \\
\hline
\end{tabular}

IDC: Infiltrating Ductal Carcinoma; ILC: Infiltrating Lobular Carcinoma; ND Not Detected

luminal and basal breast cancers display higher levels of $T A p 63$ than $\triangle N p 63$, and that $\triangle N p 63$ levels are significantly higher in basal than luminal cancer subtypes (Figure 1E). Protein analysis confirms that while luminal cells express mainly the TA isoform (indeed $\Delta \mathrm{Np} 63$ is undetectable) basal cell lines express both TA and $\Delta \mathrm{N}$ isoforms (Figure $1 \mathrm{~F})$.

We then enriched the cancer initiating population by cell sorting based on CD44/CD24 expression (Suppl. Figure 1D), and evaluated the expression levels of 
the p63 isoforms [21]. CD44 ${ }^{+}$cells, which present the characteristics of the acronym CICs, express higher mRNA levels of $\triangle N p 63$ than TAp63 despite their luminal or basal origin. Conversely, CD44- breast cancer compartment shows negligible mRNA levels of $\triangle N p 63$ and high TAp63 levels (Figure 1G). As expected, CD44 ${ }^{+}$ BCSCs showed a higher clonogenic potential than CD44BCSCs (Figure 1H).

In line with these findings, cells grown as SDACs showed a gradual increase of TAp63 expression levels and a decrease of $\triangle \mathrm{Np} 63$ levels as compared to cells grown as spheres (Suppl. Figure 1E). According to previous observations, we found that the exposure to serum dramatically reduced VIMENTIN and increased MUC1, while slightely diminished CD10 expression in basal SDACs (Suppl. Figure 1F), compared to BCSCs (Suppl. Figure 1B-1C), confirming a less stem-like phenotype in these culture conditions. These results suggest that $\Delta \mathrm{Np} 63$ may play a role in driving the tumorigenic and metastatic capacity of CD44 enriched BCSCs.

\section{$\Delta$ Np63 over-expression results in a more aggressive phenotype}

To further investigate the involvement of $\Delta \mathrm{Np} 63$ in tumorigenic potential we used lentiviral vectors to over-express TAp63, $\Delta \mathrm{Np} 63$ or silence all p63 isoforms using shRNA in both basal and luminal-derived BCSCs (Suppl. Figure 2A-2B). These cells were then injected subcutaneously into immunocompromised mice to test their ability to form tumors. Over-expression of $\Delta \mathrm{Np} 63$ resulted in increased tumor growth rate while overexpression of TAp63 reduced it in both cell types (Figure 2A). Interestingly, silencing of both isoforms completely abrogated the BCSCs ability to form tumors in vivo (Figure 2A).

Moreover, TAp63 over-expression appeared to alter BCSCs behavior allowing them to adhere in serum free medium (SFM) conditions where they normally form spheres (Suppl. Figure 2C).

In order to confirm whether p63 isoforms could regulate self-renewal potential, basal BCSCs over-expressing TAp63 and $\triangle \mathrm{Np} 63$ were serially subcutaneously transplanted in NOD/SCID mice. At the second passage, $\triangle \mathrm{Np} 63$ over-expressing cells maintain the ability to promote the formation of tumors, whose size was significantly larger than the control. Conversely, cells over-expressing TAp63 lacked the ability to establish tumors as secondary xenografts (Figure 2B).

We then investigated the role of TAp63 and $\triangle \mathrm{Np} 63$ in influencing the metastatic potential of BCSCs [29, 30]. Cells over-expressing p63 isoforms were allowed to grow in the sub-renal capsule in immunocompromised mice for up to 12 weeks. We found that TAp63 reduced the ability of basal BCSCs to give rise to metastasis, whereas luminal cells over-expressing $\Delta \mathrm{Np} 63$ gained the capacity to engraft the kidney and metastasize to distant organs such as lung (Figure 2C-2D and Suppl. Figure 2D). To ensure that metastases were generated by the distant colonization of human TAp63 and $\triangle \mathrm{Np} 63 \mathrm{BCSCs}$, previously injected in mice sub-renal capsule, we performed immunohistochemical stainings for human CK-AE (Suppl. Figure 2E). As expected, $\triangle \mathrm{Np} 63$ over-expression reduced survival rate of mice while TAp63 increased it as compared to controls (Figure 2E). All these data show that $\triangle \mathrm{Np} 63$ supports a more aggressive phenotype regardless the origin of the BCSCs, inducing increased tumor formation ability and metastatic spreading.

\section{p63 isoforms regulate EMT traits}

We tested the migration capacity of BCSCs overexpressing either TAp63 or $\triangle \mathrm{Np} 63$ using a wound healing assay, which was performed using luminal BCSCs transduced with TAp63-GFP or $\triangle \mathrm{Np63-RFP} \mathrm{lentiviral}$ vectors (or with empty vectors EV-GFP and EV-RFP). By mixing an equal number of TAp63-GFP and $\triangle$ Np63-RFP or EV-GFP and EV-RFP cells, after 24 hours we observed that, while in the control setting GFP and RFP cells are randomly interspersed, uniquely $\triangle \mathrm{Np} 63 \mathrm{BCSC}$ are confined to the migratory edge compared to TAp63 BCSCs (Figure 3A). Since a prolonged expression of high TAp63 levels could determine cell death induction, we used inducible vectors encoding for p63 isoforms (iEV, iTAp63 and $i \Delta \mathrm{Np} 63$ ). Similar results were obtained performing an invasion assay, which showed that the high expression of $\triangle \mathrm{Np} 63$ in luminal BCSCs is correlated with an enhanced invasive capacity (Figure 3B) paralleled by an increase of $\mathrm{CD}_{4} / \mathrm{CD} 24^{-}$(Suppl. Figure 2F). Conversely, expression of TAp63 in basal BCSCs significantly hampered their ability to invade (Figure 3B), reducing the mesenchymallike CD44+CD24- cell compartment (Suppl. Figure 2F). ALDH activity showed no significant variations (Suppl. Figure 2F).

In order to explore which signaling pathways involved in the metastatic process of BCSCs are regulated by p63, we analyzed the modifications of EMT-related genes by gene array analysis. While three days of $\Delta \mathrm{Np} 63$ induction generally up-regulated most of the EMT-related genes, TAp63 induction reduced the expression levels of these genes in both luminal and basal BCSCs (Figure $3 \mathrm{C})$. Interestingly, both luminal and basal BCSCs overexpressing $\triangle \mathrm{Np} 63$ showed high levels of $P D G F R B$ and TWIST1 (Figure 3C). Moreover, in basal BCSCs, $\triangle \mathrm{Np} 63$ down-regulated TFPI2 ( $>5$ fold regulation), ILIRN ( > 10 fold regulation), and ESRI ( $>10$ fold regulation) [31] and increased the mRNA expression levels of $S O X 10$ ( $>$ 12 fold regulation), NODAL ( $>3$ fold regulation), and FGFBP1 ( $>3$ fold regulation) (Figure 3C). qRT-PCR confirmed that $\triangle \mathrm{Np} 63$ significantly increased the mRNA levels of the crucial EMT-related genes TWIST, SNAIL, SLUG and VIMENTIN (Figure 3D). Though luminal and 

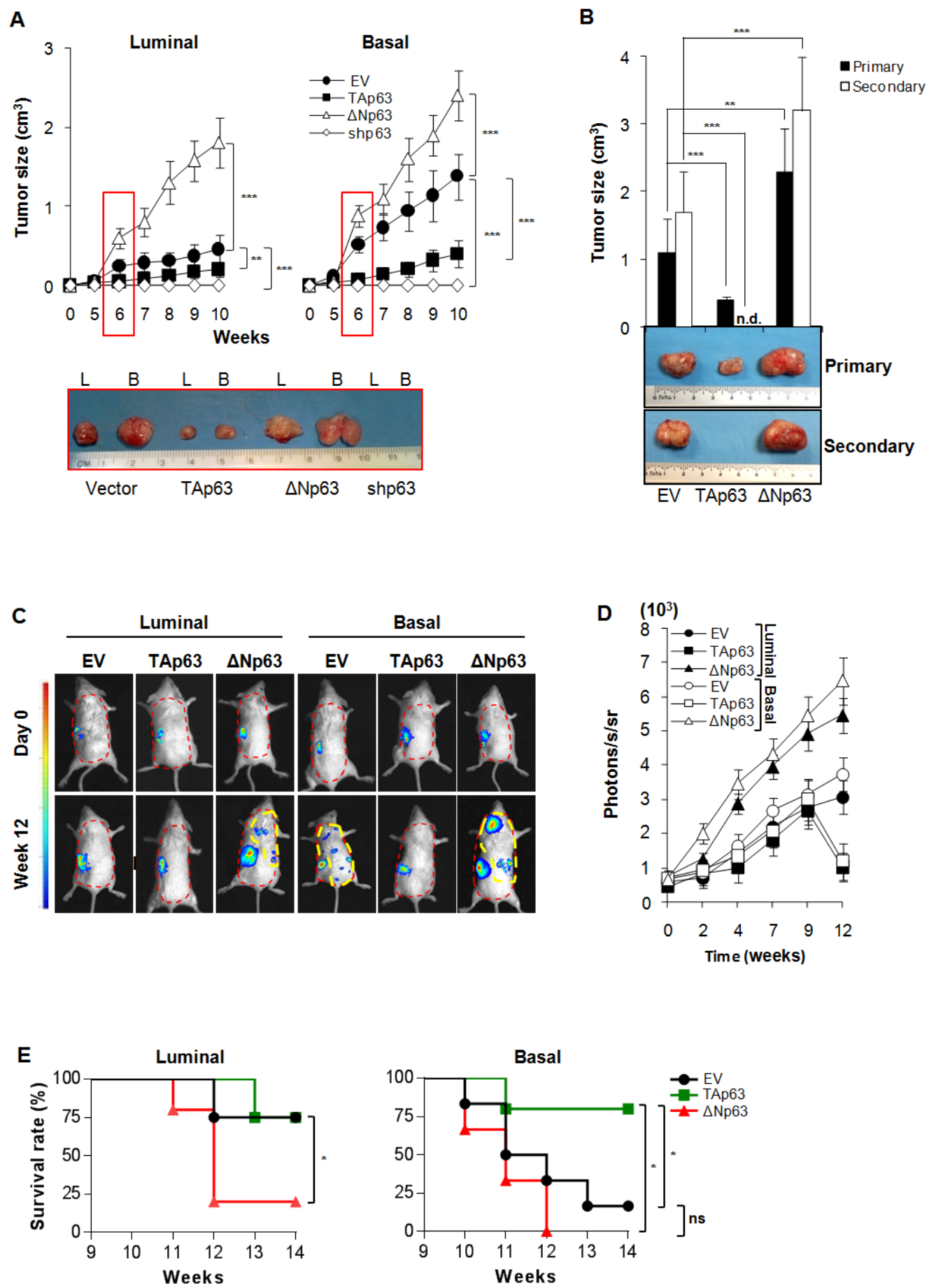

Figure 2: $\triangle$ Np63 confers metastatic potential to BCSCs. A Size of subcutaneous tumors following injection of 4,000 luminal and basal BCSCs overexpressing TAp63, $\triangle \mathrm{Np} 63$, or shp63 (empty vector, EV, was used as control). Data are represented as mean $\pm \mathrm{SD}$ of 8 tumors generated by the injection of 2 luminal and 2 basal BCSCs derived from different patients. (Lower panel) Representative gross morphology of luminal (L) and basal B tumor xenografts at 6 weeks. B Size of primary (9 weeks) and secondary (6 weeks) serial transplantation of subcutaneous tumor xenografts obtained by injection of 4,000 empty vector (EV), TAp63 and $\Delta$ Np63 derived from 2 basal BCSC primary lines. n.d. indicates: no detected tumors. C In vivo imaging analysis of sub-renal capsule xenograft tumors and metastasis growth (red dashed area) at the indicated weeks derived from the injection of 4,000 luminal and basal BCSCs transduced with empty vector (EV), TAp63 and $\triangle \mathrm{Np} 63$. D In vivo imaging kinetics analysis as in $\mathbf{C}$ at the indicated time points. Data represent mean $\pm \mathrm{SD}$ of 3 independent experiments performed with 2 luminal and 2 basal BCSCs derived from different patients. E Survival rate percentage of mice treated as in $\mathbf{C}$, at the indicated weeks. Results were analyzed using the log-rank test. Photons $/ \mathrm{seconds} / \mathrm{steradian}(\mathrm{photons} / \mathrm{s} / \mathrm{sr})=4$ for all the metastatic lesions (yellow dashed area) was chosen as limit to proceed with mice sacrifice. * indicates $P<0.05$, ** indicate $P<0.01$ and $* * *$ indicate $P<0.001$. ns indicates non statistically significant. 
A

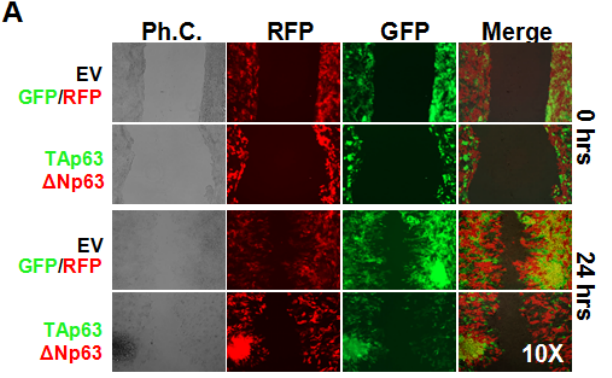

C

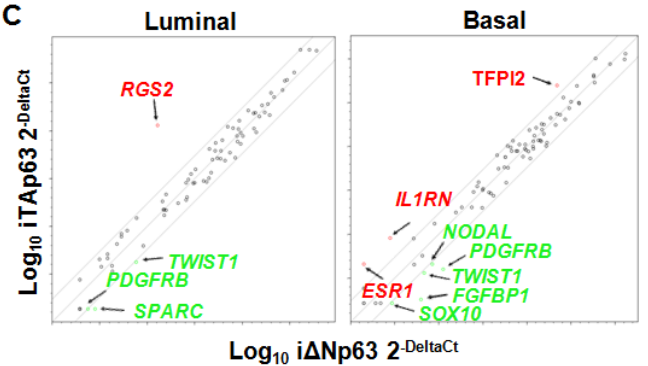

E
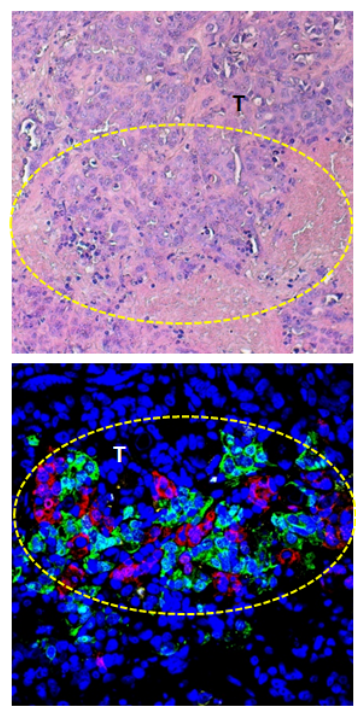

EVIEV

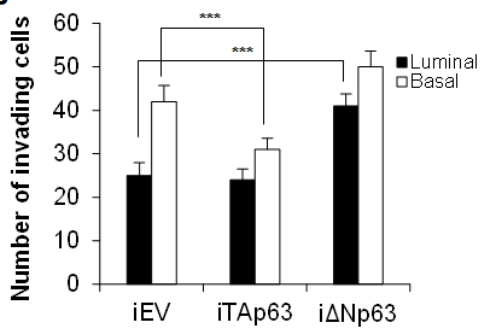

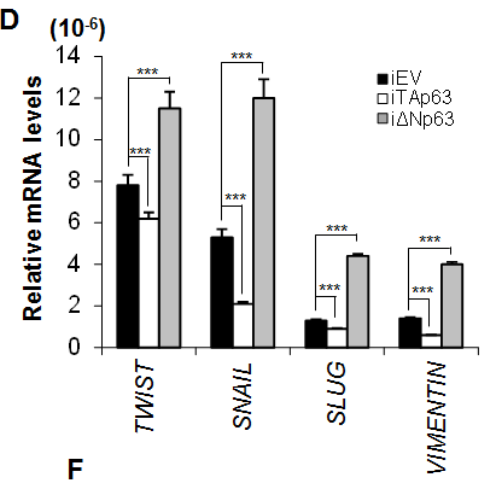

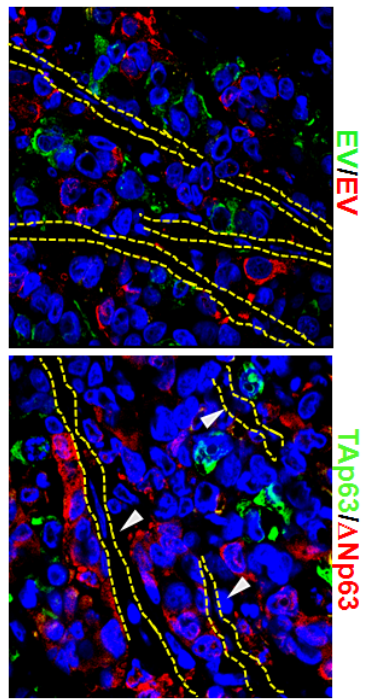

Figure 3: EMT program is regulated by $\Delta$ Np63 expression. A. Representative wound healing analysis of 3 independent experiments performed with 3 luminal BCSCs over-expressing TAp63-GFP (green color) or $\triangle \mathrm{Np} 63$-RFP (red color). Cell migration was monitored for $24 \mathrm{~h}$. B. Invasion assay performed at 72 hours with luminal and basal iEV, iTAp63 or i $\triangle$ Np63 BCSCs following 5 days of exposure to doxycycline. Data are represented as mean \pm S.D. of 3 independent experiments derived from 3 luminal and 2 basal BCSC lines. C. EMT-related gene expression analysis of luminal and basal iEV, iTAp63 or i $\triangle \mathrm{Np} 63$ BCSCs following 3 days of exposure to doxycycline. Fold variation of $\triangle \mathrm{Np} 63$ vs TAp63 $\geq 3$ are shown in green or red. D. TWIST, SNAIL, SLUG and VIMENTIN mRNA expression levels in luminal BCSCs as in C. Results show mean \pm S.D. of 3 independent experiments. E. (Upper panels) Representative H\&E staining performed on paraffin-embedded sections of xenografts generated by subcutaneous injection of 1:1 mixture of EV-GFP:EV-RFP or TAp63GFP: $\triangle N p 63-R F P$ luminal BCSCs. (Lower panels) Immunofluorescence analysis of TAp63-GFP (green color) or $\triangle \mathrm{Np63-RFP} \mathrm{(red} \mathrm{color)}$ performed on paraffin-embedded sections of xenografts generated as in $\mathbf{E}$ Dashed yellow circles represent tumor growth areas (T). White arrowheads indicate tumor buds overexpressing $\triangle \mathrm{Np} 63$-RFP (red color) at the tumor invasion front. F. Representative immunofluorescence analysis of TAp63-GFP (green color) or $\triangle \mathrm{Np} 63$-RFP (red color) performed on paraffin-embedded sections of xenografts generated as in E White arrowheads and dashed lines indicate vessels. * indicates $P<0.05$, ** indicate $P<0.01$ and *** indicate $P<0.001$. ns indicates non statistically significant. 
basal BCSCs showed different combinations of activated/ repressed transcription factors and signaling pathways, these data indicate that the induction of different p63 isoforms influences their migratory/invasive behaviour. Finally, using luminal BCSCs transduced with TAp63GFP or $\triangle N p 63-R F P$ lentiviral vectors we tested in vivo localization of TAp63 and $\triangle \mathrm{Np} 63$ cells in tumors. Interestingly, xenografts generated by the injection of a 1:1 ratio of TAp63-GFP/ $\triangle \mathrm{Np} 63-\mathrm{RFP}$ luminal BCSCs showed that cells over-expressing $\Delta \mathrm{Np} 63$ were located as tumor buds at the invasive front (Figure $3 \mathrm{E}$ ). Strikingly, $\Delta \mathrm{Np} 63$ over-expressing cells were localized in close proximity to endothelial cells suggesting their migratory aptitude (Figure $3 \mathrm{~F}$ ). Hence, $\Delta \mathrm{Np} 63$ might play a key role in EMT induction and in the invasive potential of BCSCs under the influence of tumor microenvironment.

\section{$\Delta \mathrm{Np} 63$ induces CD44v6 expression}

Tumor associated cytokines are known to enhance cell migration and invasion [32-35]. By analyzing the most common tumor microenvironmental cytokines, we observed that basal BCSCs, as well as cancer-associated fibroblasts (CAFs) isolated from a basal cancer sample, secrete hepatocyte growth factor (HGF), stromal cellderived factor 1 (SDF-1) and osteopontin (OPN) in the medium (Figure 4A). Exposure to SDF-1, OPN, or CAFconditioned medium (CAF-CM) markedly increased the expression levels of $\triangle N p 63$ in both luminal and basal BCSCs (Figure 4B). $\triangle \mathrm{Np} 63$ regulates the expression of CD44 splicing isoforms in head and neck cancer [32]. Moreover, we have recently demonstrated that tumor niche is an extraordinary source of microenvironmental stimuli that induce CD44v6 expression, leading to the reprogramming of CD44v6 progenitors in metastatic $\mathrm{CD}_{4} \mathrm{v}^{+}{ }^{+}$stem cells [22]. In this context, we explored whether p63 isoforms could regulate CD44 isoforms expression also in breast cancer. Indeed, CD44 has been amply reported as a marker that identifies breast cancer cells with a pronounced self-renewal and metastatic potential [33] and, interestingly, circulating tumor cells endowed with metastatic capabilities have been shown to highly express the CD44 isoform variant, CD44v6 [34].

To investigate if CD44v6 has a functional role on BCSCs and whether it is differently modulated by p63 isoforms, we firstly tested CD44v6 expression on iTA/ i $\triangle$ Np63 BCSCs. i $\triangle N p 63$ BCSCs express significantly higher CD44v6 levels than iTAp63 BCSCs (Figure 4C). Moreover, the over-expression of $\triangle \mathrm{Np} 63$ in luminal BCSCs enforced CD44v6 expression while it was constant in basal BCSCs, which already harbour high level of CD44v6 (Figure 4C).

In colorectal progenitor cells, CD44v6 is boosted by the secretion of OPN, SDF-1, HGF microenvironmental cytokines [22]. Thus, we tested whether CAF-CM could affect CD44v6 expression in BCSCs. We found that luminal BCSCs treated with CAF-CM increased CD44v6 expression levels (Figure 4D), likely for an increase in $\Delta \mathrm{Np} 63$ isoform.

To demonstrate the presence of a functional link between p63 isoforms and CD44v6 in BCSC behaviour, we performed a clonogenic, proliferation and invasion assay on iTAp63 basal and i $\triangle \mathrm{Np} 63$ luminal BCSCs transduced with lentiviral vectors encoding for CD44v6 (v6) or shCD44v6 (shv6), respectively. Since luminal BCSCs harbor lower levels of $\triangle \mathrm{Np} 63$ than basal ones (Figure 1F), CD44v6 was down-regulated in luminal BCSCs over-expressing $\Delta \mathrm{Np} 63$ (i $\Delta \mathrm{Np} 63 /$ shv6) and upregulated in the basal subtype over-expressing TAp63 (iTAp63/v6) (Figure 4E-4G). In brief, BCSCs were initially transduced with iEV, iTAP63 or i $\triangle \mathrm{Np} 63$, and after 7 days with vectors coding for v6 or shv6 and their controls, EV and Scr respectively. After one week, cells were exposed to doxycycline for 5 days (to induce expression of the p63 isoforms). While the downregulation of CD44v6 blunted the clonogenic, proliferative and invasive capability of luminal BCSCs bearing i $\triangle \mathrm{Np} 63$, over-expression of CD44v6 restored these features in basal BCSCs transduced with iTAp63 (Figure 4E-4G).

\section{$\Delta$ Np63 enhances PI3K/AKT pathway activity controlling BCSCs chemoresistance}

CD44v6 is essential for colorectal CSC migration and metastatic outgrowth and its expression is sustained by $\mathrm{PI} 3 \mathrm{~K} / \mathrm{AKT}$ pathway [22]. Indeed, PI3K is involved in multiple cellular events and is required for proliferation and migration of different cancer cell types including breast cancer cells $[22,35]$. Starting from the observation that in our model CD44v6 expression is augmented in luminal BCSCs following the over-expression of $\triangle \mathrm{Np} 63$ (Figure 4C) we sought to explore whether p63 could modulate the activation of the PI3K/AKT pathway. Western Blot analyses showed that exogenous expression of TAp63 reduced $\mathrm{PI} 3 \mathrm{~K} / \mathrm{AKT}$ pathway activation, as shown by a considerable decrease in AKT phosphorylation (Figure 5A-5B). Conversely, $\triangle \mathrm{Np} 63$ over-expression strongly boosted this signalling pathway (Figure 5A-5B). Notably, whereas a similar reduction in p-AKT expression level is observed in luminal and basal TAp63 over-expressing cells, over-expression of $\Delta \mathrm{Np} 63$ led to a more pronounced phosphorylation of AKT in basal cells as compared to luminal ones (Figure 5A-5B). In line with the observation that $\triangle \mathrm{Np} 63$ could promote a proliferative phenotype through the regulation of the $\mathrm{PI} 3 \mathrm{~K} / \mathrm{AKT}$ pathway, we administered in vitro the PI3K inhibitor (BKM120) in combination with either a nonsteroidal aromatase inhibitor (Anastrozole) or a member of the taxane drug class (Docetaxel) (Figure 5C). Indeed, aromatase inhibitors in combination with PI3K inhibitors are currently undergoing clinical trials in order to 

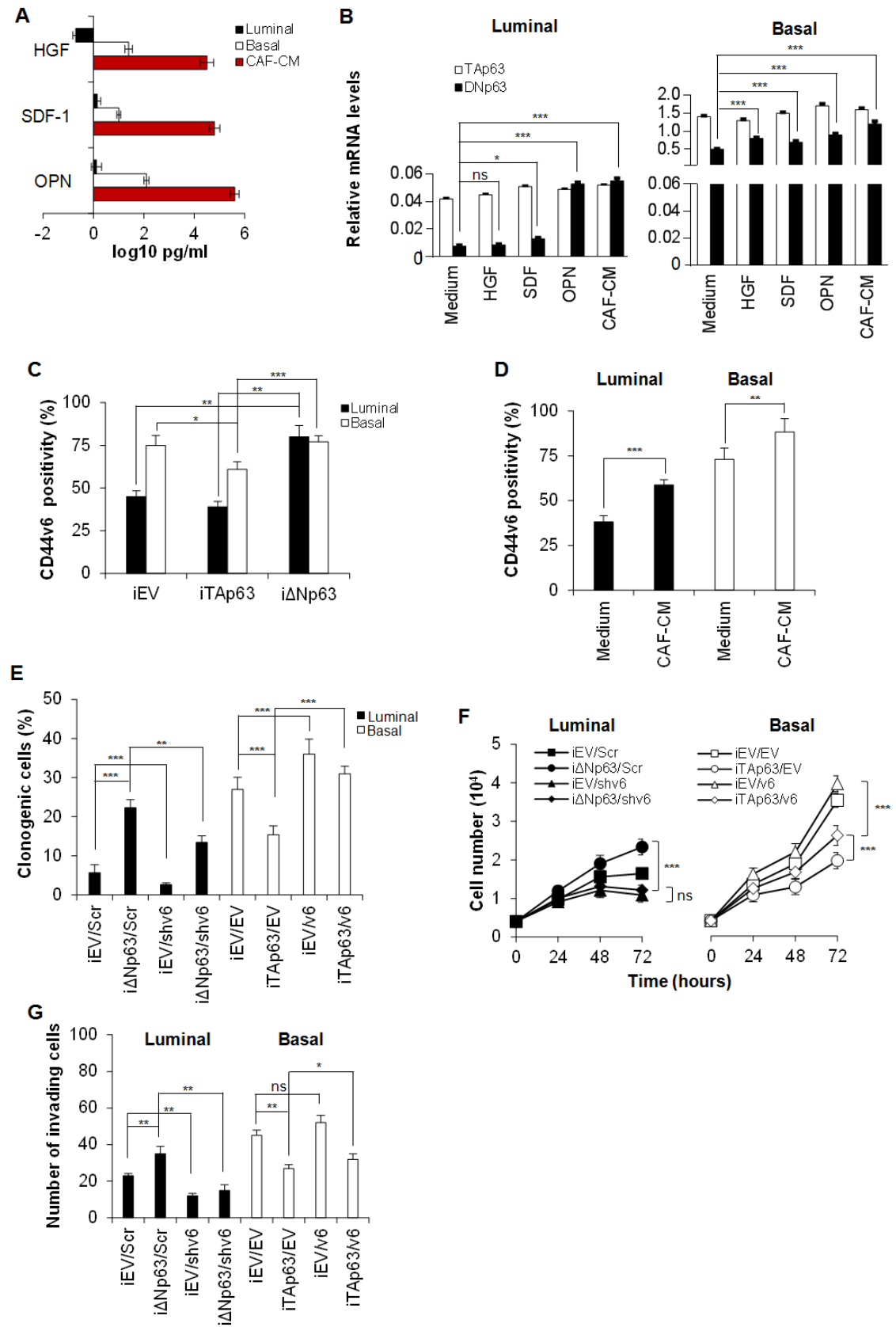

Figure 4 : $\Delta$ Np63 enhances CD44v6 expression. A. HGF, SDF-1 and OPN levels in supernatants of luminal and basal BCSCs, and cancer associated fibroblasts (CAFs) freshly isolated from a basal breast tumor sample. Data are mean \pm SD of 3 independent experiments performed with 3 luminal and 2 basal BCSC lines derived from different patients. B. TAp63 and $\triangle N p 63$ mRNA expression levels in luminal and basal BCSCs, exposed to HGF, SDF-1 and OPN or CAF-conditioned medium (CAF-CM) for 24 hours. GAPDH amplification was used as endogenous control. Results show mean $\pm \mathrm{SD}$ of 3 independent experiments performed with 3 luminal and 2 basal BCSC primary lines. C. Percentage of CD44v6 positive cells in luminal and basal iEV, iTAp63 or i $\triangle \mathrm{Np} 63 \mathrm{BCSCs}$ performed by flow cytometry. Data are shown as mean \pm SD of 3 independent experiments using 3 luminal and 2 basal BCSC primary lines. D. CD44v6 positivity percentage in luminal and basal BCSCs treated with medium (Medium) or CAF-CM for 24 hours. Results are mean \pm SD of 3 independent experiments accomplished using 3 luminal and 2 basal BCSCs derived from different patients. E. Percentage of clonogenic luminal BCSCs transduced with either or both $\mathrm{i} \triangle \mathrm{Np} 63$ and shCD44v6 (shv6) vector and their control, iEV and Scr respectively. Basal BCSCs were transduced with either or both iTAp63 and CD44v6 (v6) expressing vectors and their iEV/EV controls. BCSCs were firstly transduced with the inducible isoforms of p63 and after one week with shv6 and v6. After 7 days, cells were exposed to doxycycline for 5 days before the experiment and plated in doxycycline containing medium for the entire assay. Results are mean \pm SD of 3 independent experiments performed with 3 luminal and 2 basal BCSC primary lines. F. Cell number analysis of luminal and basal BCSCs as in E, up to 72 hours. G. Invasion assay at 72 hours of luminal and basal BCSCs treated as in E. Results are mean \pm SD of 4 independent experiments. * indicates $P<0.05, * *$ indicate $P<0.01$ and $* * *$ indicate $P<0.001$. ns indicates non statistically significant. 

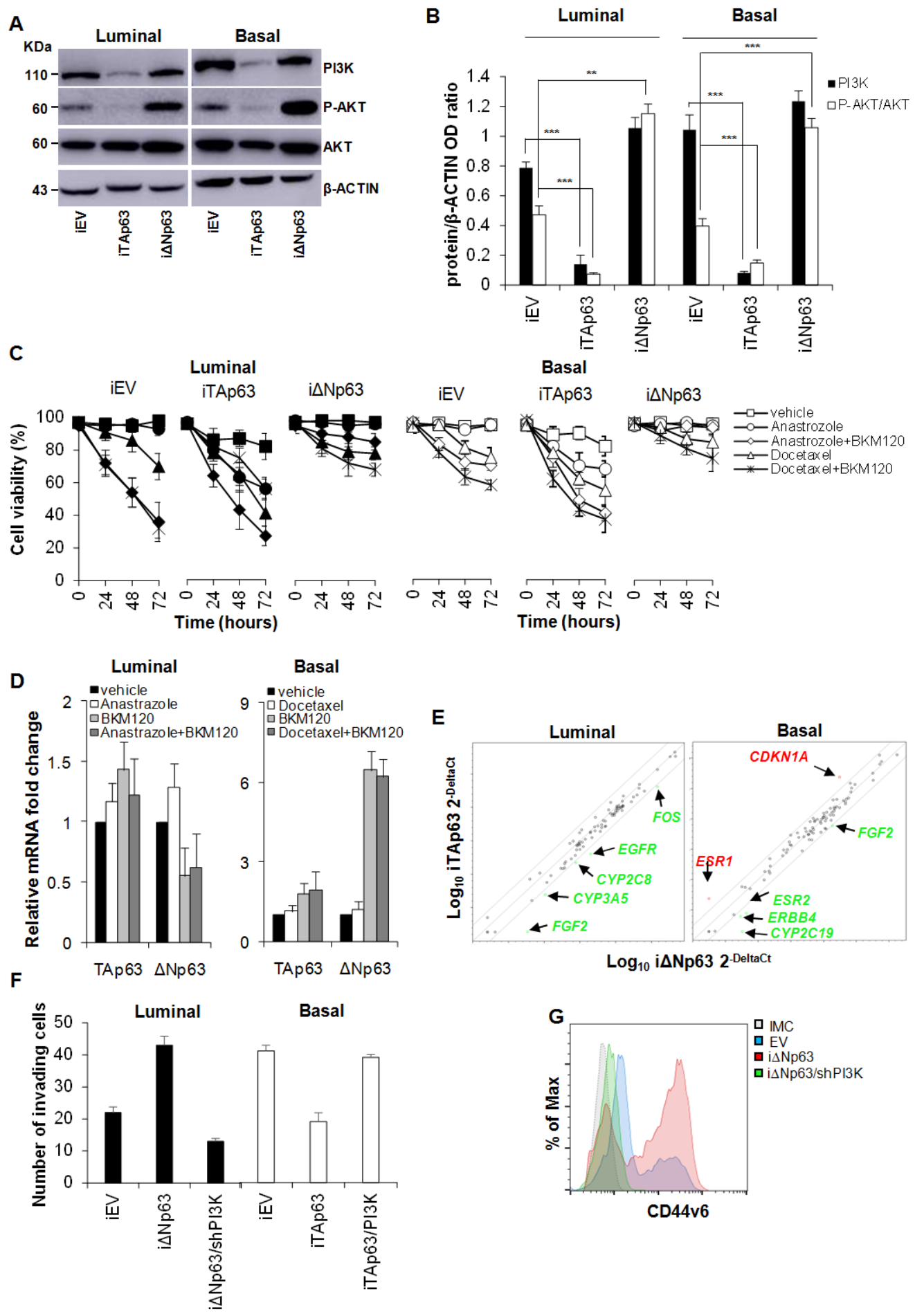

Figure 5: TAp63 sensitizes BCSCs to therapeutic drugs. A. Immunoblot analysis of PI3K, P-AKT and AKT, in luminal and basal BCSCs transduced with doxycycline inducible EV, TAp63 or $\triangle$ Np63 (iEV, iTAp63 and i $\triangle$ Np63). $\beta$-ACTIN was used as loading control. B. Relative expression levels of PI3K and P-AKT/AKT as in A. Data are expressed as mean \pm SD of 3 independent experiments. C. Cell viability analysis of BCSCs transduced as in A and treated with Anastrozole or Docetaxel, alone or in combination with BKM120 up to 72 hours. Data are mean \pm SD of 3 independent experiments. D. Relative TAp63 and $\triangle$ Np63 expression in luminal and basal BCSCs that survived to the above mentioned treatments. Data are expressed as mean $\pm \mathrm{SD}$ of 3 independent experiments performed in 3 luminal and 2 basal BCSC lines. E. Scatter plot of drug-resistance related genes in luminal and basal BCSCs as in A. Genes indicated in green and red show fold variation $(\Delta \mathrm{Np} 63$ vs TAp63) $\geq 3$. F. Invasion assay of luminal iEV, i $\Delta \mathrm{Np} 63$ or i $\Delta \mathrm{Np} 63 / \mathrm{shPI} 3 \mathrm{~K}$ and basal iEV, iTAp63 or iTAp63/ PI3K performed at 72 hours. Data are represented as mean \pm SD of 3 independent experiments using 3 luminal and 3 basal BCSCs. G. Flow cytometry analysis of CD44v6 in luminal BCSCs as in F. * indicates $P<0.05$, ** indicate $P<0.01$ and *** indicate $P<0.001$. ns indicates non statistically significant. 
overcome refractoriness to standard anti-tumoral therapies in estrogen receptor-positive (luminal) breast cancers [36]. Moreover, PI3K inhibitors in combination with taxanes has shown to enforce the effectiveness of taxanes in both estrogen receptor-positive and -negative breast cancers [37].

Interestingly, i $\triangle \mathrm{Np} 63$ rendered luminal BCSCs more refractory to the administration of either BKM120+Anastrozole or BKM120+Docetaxel (Figure $5 \mathrm{C}$, left panel). On the other hand, iTAp63 sensitizes basal BCSCs to the above-mentioned single and combinatorial therapeutic regimens (Figure 5C, left panel). We then analyzed $\Delta \mathrm{Np} 63$ and TAp63 expression in the untrasduced BCSCs survived to the anti-tumor treatments. Interestingly, whereas TA expression levels were not markedly modulated upon treatments in both BCSC subtypes, $\triangle \mathrm{Np} 63$ expression was significally upregulated in basal BCSCs (Figure 5D), suggesting an involvement of $\triangle \mathrm{Np} 63$ in drug resistance at least in this molecular subtype. Gene expression profile of a panel of effectors commonly involved in drug resistance/ susceptibility revealed that $\mathrm{i} \Delta \mathrm{Np} 63$ luminal cells showed high expression levels of drug resistance-related markers such as FOS, EGFR, CYP2C8,CYP3A5 and FGF2 (Figure 5E). Conversely, basal iTAp63 BCSCs showed an enrichment in CDKN1A (p21) [38] and ESR1 (encoding for the estrogen receptor-alpha, ER- $\alpha$ ) (Figure 5E). These data clearly suggest that TAp63 isoform plays a fundamental role in making aggressive breast cancer phenotypes more sensitive to hormone therapy, likely making them dependent on estrogen pathway through the up-regulation of ER- $\alpha$ in basal cancer. Significant change in ER- $\alpha$ expression was not observed in luminal BCSCs over-expressing $\Delta$ Np63 (Suppl. Figure $2 \mathrm{G}$ ), indicating that cell refractoriness as well as tumorigenic and metastatic potential acquired by these cells is independent on ESR1 expression levels. Moreover, in luminal BCSCs overexpressing $\Delta \mathrm{Np} 63$ the silencing of $\mathrm{PI} 3 \mathrm{~K}$ (i $\Delta \mathrm{Np} 63 / \mathrm{shPI} 3 \mathrm{~K}$ ) hampered their invasion capacity whereas the invasive potential of basal TAp63 BCSCs was restored upon PI3K over-expression (iTAp63/PI3K) (Figure 5F). Of note, PI3K silencing highly reduced the expression of CD44v6 overcoming the effect of $\Delta \mathrm{Np} 63$ (Figure $5 \mathrm{G}$ ). These data confirmed that p63 isoforms orchestrate breast cancer progression through the activation of $\mathrm{PI} 3 \mathrm{~K} / \mathrm{CD} 44 \mathrm{v} 6$ axis (Suppl. Figure 3).

\section{DISCUSSION}

The presence, role and characterization of the cells with tumorigenic and metastatic potential comprised in breast cancer is a matter of intense research [39, 40]. Here, we show that BCSCs, which express $\triangle \mathrm{Np} 63$, are endowed with an intrinsic heightened invasive and metastatic activity that can be reverted to a phenotype with abrogated invasive potential by switching to the TAp63 expression.
Different groups have shown a moderate to intense p63 nuclear expression exclusively in the mammary gland's basal layer (where stem cells are located), with no detectable expression in the luminal cells (containing progenitor and differentiated cells) that line the glandular lumen [11]. In particular, basal cells were found enriched with $\Delta \mathrm{Np} 63$, in contrast with the TAp63 expression that was restricted to the luminal epithelial glandular cells [10]. According to the current model, which describes CICs as derived from normal stem cells, it was shown that in tumor tissues, $\Delta \mathrm{Np} 63$ was almost absent and confined to only some myoepithelial and basal cells, whilst most of the cells express the TAp63 protein. Different models were recently suggested with regard to $\Delta \mathrm{Np} 63$ role in breast tumor progression. It was recently reported that $\Delta \mathrm{Np} 63$ controls breast cancer cells migration and invasion [30, 41] and increases their tumor initiating activity [28, 29].

Our results extend these findings identifying a novel pathway regulated by p63 isoforms and highlighting also a role for the TAp63 isoforms.

Our findings show that BCSCs appear to express both $\mathrm{TA}$ and $\triangle \mathrm{Np} 63$ and that $\triangle \mathrm{Np} 63$ expression is lost when cancer cells develop a more differentiated phenotype. As expected $\Delta \mathrm{Np} 63$ expressing cells have a more stem phenotype and appear to have a higher tumorigenic and metastatic potential.

Different studies in recent years have shown the $\triangle \mathrm{Np} 63$ key role in the induction of EMT in breast cancers [42]. EMT has been described as a crucial step in tumor initiation and acquisition of metastatic traits $[33,43]$. In line with these findings, we observe that $\Delta \mathrm{Np} 63$ enhances the clonogenic potential and sustains an EMT programme mainly inducing the expression of TWIST and PDGFRB. The ability of TAp63 to delay the growth of primary tumors and abrogate metastasis formation, improving the survival rate of mice bearing tumors, may be related to the promotion of an epithelial phenotype observed in basal TAp63 BCSCs. The weak tumorigenic potential of luminal $\mathrm{BCSC}$ sould be attributed to the presence of a small cell subset expressing $\Delta \mathrm{Np} 63$.

We also show that TA and $\Delta \mathrm{Np} 63$ isoforms exert their opposite effects at least in part through the opposite regulation of a $\mathrm{PI} 3 \mathrm{~K} / \mathrm{CD} 44 \mathrm{v} 6$ pathway. Indeed, while $\Delta \mathrm{Np} 63$ expression results in activation of the PI3K/AKT pathway leading to CD44v6 up-regulation and a more aggressive mesenchymal phenotype, TA expression has opposite effects. Blocking PI3K activation by means of inhibitors or silencing of CD44v6 reduces the ability of $\Delta \mathrm{Np} 63$ to induce a more tumorigenic and invasive phenotype demonstrating the importance of this pathway. Clearly as reported in the literature [28-30, 41] this is not the only pathway controlled by p63 that appears more and more to be a master gene regulating cancer cell differentiation as it does in normal epithelial cells. Our data also show that the subset of cells that over-expresses $\Delta \mathrm{Np} 63$ (presumably the cancer initiating subpopulation) 
are also as expected more resistant to current therapies but can be sensitized using PI3K inhibitors, suggesting that the use of these inhibitors in combination with currently used therapies could be an effective strategy to overcome resistance, prevent relapse and metastatic spreading.

\section{MATERIALS AND METHODS}

\section{Tissue collection, isolation and culture of cancer cells}

Breast cancer tissues were collected at the Department of Surgical and Oncological Sciences in accordance with the ethical standards of the University of Palermo institutional committee, and then characterized by tumor type, grading, or ER/PR/HER-2/KI67/p63 expression. Normal breast tissue was obtained from the histologically uninvolved resection. Breast tissues were mechanically and enzymatically digested using collagenase (1.5 mg/mL; Gibco) and hyaluronidase (20 mg/mL; Sigma Aldrich) in Dulbecco's Modified Eagle Medium (Gibco), for 1 hour at $37^{\circ} \mathrm{C}$. Cells were maintained in culture in serum-free medium (SFM), in presence of fibroblast growth factor (bFGF; $10 \mathrm{ng}$ / $\mathrm{mL}$ ) and epidermal growth factor (EGF, $20 \mathrm{ng} / \mathrm{mL}$ ) in ultra-low attachment flasks (Corning, Lowell, MA) as previously described [44]. These culture conditions make cells growing as aggregates conventionally defined as spheres. Weekly, spheres are enzymatically dissociated with accutase (Gibco) and plated in fresh SFM. The in vitro model adopted to obtain the non tumorigenic spherederived adherent cells (SDACs), is based on dissociation of BCSCs and subsequent culture in Dulbecco's modified Eagle medium (DMEM) supplemented with 10\% FCS in adherent conditions for at least 25 days. Cancer associated fibroblasts (CAFs) were isolated, by enzymatic digestion, from basal breast cancer samples and cultured in presence of DMEM plus $10 \%$ FBS in adherent conditions.

\section{Cell proliferation}

Cell proliferation was assessed using CellTiter $96^{\circledR}$ AQueous One Solution Cell Proliferation Assay kit (MTS) according to the manufacturer's instruction.

The in vitro sensitivity assay was performed by plating 250 cells in ultra-low attachment 96-well plates (Corning) and exposing them to vehicle, $50 \mu \mathrm{M}$ Anastrozole (Selleckchem), or 100nM Docetaxel (Selleckchem), alone or in combination with $5 \mu \mathrm{M}$ BKM120 (NVP-BKM120, Selleckchem). Cell viability was measured for up to $72 \mathrm{hrs}$, using the CellTiterGlo $^{\circledR}$ Luminescent Cell Viability Assay kit (Promega) according to the manufacturer's instruction. The results were analyzed by using Infinite ${ }^{\circledR}$ F500 (Tecan). Each combinatorial regimen was evaluated in triplicate.

\section{Clonogenenic assay}

The clonogenic capacity of BCSCs was assayed by dissociating breast cancer spheroids and plating them 1 cell/well in ultra-low attachment 96 well microplates with flat bottom (Corning, Lowell, MA), using a FACS Aria I. Results were statistically evaluated after 4 weeks.

\section{Migration and invasion assay}

Transduced and control BCSCs were seeded in adherent conditions in six-well plates at a density of $1 \times 10^{6}$ cells/well and cultured to achieve a confluent cell layer in culture medium. The day after a sterile $200 \mu \mathrm{l}$ pipette tip was used to straight scratch a constant-diameter stripe in the confluent cell monolayer. Plates were then washed with $1 \mathrm{ml}$ of PBS to remove detached cells and debris and the medium was replaced using a fresh culture medium. Wound healing was monitored, by EVOS ${ }^{\mathrm{TM}} \mathrm{fl}$ Digital Inverted Fluorescence Microscope, following the migrating cells in the gap during a period of 24 hours postscratch.

Cells tested for invasive potential $\left(2 \times 10^{3}\right)$ were plated into Matrigel-coated (BD Biosciences) transwells of $8 \mu \mathrm{m}$ pore size (Corning). DMEM supplemented with $10 \%$ human serum (Euroclone, ECS0219D) (600 $\mu \mathrm{l} /$ well) was used as chemo-attractant in the lower part of transwells. Migration was calculated by the microscopic observation of cells migrated in the lower part of transwell up to $72 \mathrm{hrs}$.

\section{Flow cytometry/cell sorting}

Cells were stained with conjugated CD44-PE (G44-26, mouse, IgG2b, BD), CD24-APC (ML5, mouse, IgG2a, R\&D), CD44v6-APC (2F10, mouse, IgG1, R\&D) antibodies or matching isotype controls. Samples were acquired and analyzed by Accuri (BD Biosciences) flow cytometer. FACS sorting, using a FACS aria (BD Biosciences), was performed on cells stained with CD44PE and CD24-APC, whose quality was monitored by flow cytometry. ALDH activity was measured by using ALDEFLUOR assay (Stem Cell technologies). All data were analyzed using FlowJo software (Tree Star). Sorted cells were collected and used for $\Delta \mathrm{Np} 63$ and TAp63 mRNA expression.

\section{Immunohistochemistry/Immunofluorescence}

For antigen unmasking, five- $\mu$ m-thick paraffinembedded sections of basal and luminal breast cancer tissues, such as their normal counterpart, were heated 
with a retrieval solution in $10 \mathrm{mM}$ sodium citrate $(\mathrm{pH}$ 6.0). After rising in $\mathrm{dH}_{2} \mathrm{O}$, endogenous peroxidase was inhibited by incubation of 5 minutes with $3 \% \mathrm{H}_{2} \mathrm{O}_{2}$. Cells were permeabilized with PBS plus $0.1 \%$ Triton X-100 (TBS) for 10 minutes on ice. Then cells were exposed to specific antibodies directed against ER (6F11, mouse IgG1 Leica), PR (1E2, rabbit IgG, Roche), HER-2 (D8F12, rabbit IgG, CST), CK5 (XM26, mouse $\operatorname{IgG}_{1 \mathrm{k}}$, Leica),

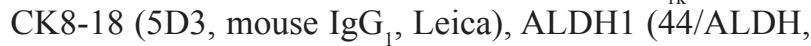
mouse $\left.\operatorname{IgG}_{1}, \mathrm{BD}\right)$, p63 (4A4, mouse $\mathrm{IgG}_{2 \mathrm{a}}$, Santa Cruz), CK-AE (AE1/AE3, mouse $\operatorname{IgG}_{1}$, Novocastra), or isotype matched controls at appropriate dilutions overnight at $4^{\circ} \mathrm{C}$. Sections were then incubated with biotinylated immunoglobulins, washed and following exposed to streptavidin. Stainings were revealed using 3-amino-9ethylcarbanzole (AEC, Dako) substrate and counterstained with aqueous hematoxylin.

$\mathrm{H} \& \mathrm{E}$ staining was performed using standard protocols on $5-\mu \mathrm{m}$ paraffin sections.

Immunofluorescence was performed on cytospun cancer sphere cells or SDACs cultured on round coverslips into 24 well plates, fixed with $2 \%$ paraformaldehyde for 20 minutes at $37^{\circ} \mathrm{C}$ or on five- $\mu \mathrm{m}$-thick paraffin-embedded sections. After permeabilization, cells were stained overnight at $4^{\circ} \mathrm{C}$ using antibodies against ER, PR, HER2, CK5, CK8-18, CK14, MUC1 (HMPV, mouse IgG ${ }_{1 \mathrm{k}}$, BD Biosciences), CD10 (FR4D11, mouse $\mathrm{IgG}_{1}$, Santa Cruz), VIMENTIN (R28, rabbit, CST), p63, GFP (D5.1, rabbit IgG, CST), RFP (ab62341, rabbit IgG, Abcam), or isotype-matched controls at appropriate dilutions. Then, cells were labelled with Alexa Fluor 488- or Rhodamine red-conjugated secondary antibodies (Thermo Fisher Scientific). Nucleus counterstaining was performed using Toto-3 iodide (RNAse treatment was performed to prevent the cytoplasmic RNA staining). Samples were analyzed using a confocal microscope (Nikon D-Eclipse C1).

\section{RNA extraction and real-time PCR}

Breast cancer tissues and BCSCs were used to isolate RNA using the RNeasy Plus Mini Kit (Qiagen). Total RNA was retro-transcribed using the High-Capacity c-DNA Reverse Transcription kit (Applied Biosystem). Quantitative real-time PCR analysis was performed using a SYBR Green master mix containing the following primers: TAp63 F-ATTGTTCTCCGTTCGTTG; TAp63 R-GATGTAAGGGTCAGGGCAG; $\triangle N p 63 \quad$ F-TATTGTAAGGGTCTCGGG; $\quad \Delta N p 63$ R-GGCATTGTTTTCCAGGTA; GAPDH F-GCTTCGCTCTCTGCTCCTCCTGT; GAPDH R-TACGACCAAATCCGTTGACTCCG. The Taqman master mix was prepared with the following primers: CD44v6 Hs_01075870; PI3K Hs00907954_m1; GAPDH 4352934-1107035 (Applied Biosystem). The relative quantitation of gene expression was calculated on triplicate reactions using the comparative $\mathrm{Ct}$ method $(\triangle \triangle \mathrm{Ct}) . G A P D H$ was used as housekeeping gene.

For the gene expression arrays, RNA samples were retro-transcribed ( $\mathrm{RT}^{2}$ First Strand Kit, Qiagen) and real time PCR were performed using the $\mathrm{RT}^{2}$ Profiler PCR Arrays Human Epithelial to Mesenchymal Transition (PAHS-090ZR) and Human Cancer Drug Resistance (PAHS-004ZR). Collected data were analyzed with the Qiagen $\mathrm{RT}^{2}$ Profiler PCR Arrays data analysis software.

\section{Western blot}

BCSCs were collected, washed twice in ice-cold PBS and then resuspended in ice-cold buffer containing TPER Reagent (Pierce) $300 \mathrm{mM} \mathrm{NaCl}$ (Sigma Aldrich) $1 \mathrm{mM}$ orthovanadate (Sigma Aldrich) $2 \mathrm{mM}$ pefabloc (Roche), proteinase inhibitor cocktail (aprotinin, pepstatin and leupeptin, each at $5 \mu \mathrm{g} / \mathrm{mL}$, Sigma Aldrich) and incubated for $30 \mathrm{~min}$ on ice. Protein extracts were resolved using a SDS-PAGE gel and separated by electrophoresis, transferred to nitrocellulose membranes (Hybond-C Extra, Nitrocellulose, Amersham) and subsequently blocked with PBS Tween 20 0.1\% (Sigma Aldrich) not-fat dry milk 5\% (Sigma-Aldrich) for 1 hour at R.T. Immunodetection was performed by incubating the membranes with the primary antibodies at $4{ }^{\circ} \mathrm{C}$ O.N., and finally with secondary antibodies for 1 hour at R.T. The following antibodies were used: p63- $\alpha$ (D2K8X, rabbit IgG, CST), p63 (4A4, mouse IgG2A, Santacruz), p110- $\alpha$ (PI3K, C73F8, rabbit IgG, CST), AKT (rabbit, CST \#9272), P-AKT (D9E, rabbit, IgG, CST), $\beta$-ACTIN (8H10D10, mouse $\mathrm{IgG}_{2 \mathrm{~b}}, \mathrm{CST}$ ), anti-mouse HRP-conjugated (goat IgG, ThermoFisher Scientific), anti-rabbit HRP-conjugated (goat IgG, ThermoFisher Scientific). Immunoreactive bands were detected using SuperSignal ${ }^{\text {TM }}$ West Dura Extended Duration Substrate (ThermoFisher Scientific) and revealed by using the Amersham imager 600 (GE Healthcare).

\section{Production of lentiviral particles and BCSCs transduction}

Gene transfer of luciferase (LUC) and green fluorescent protein (GFP) was assessed using the p-TWEEN lentiviral vector. The synthetic genes encoding $\Delta$ Np63- $\alpha$ (Addgene \#26979) and TAp63- $\gamma$ (Addgene \#26977) were inserted into the p-TWEEN EGFP and p-TWEEN RFP lentiviral expression vector, and in the pInducer20 Tet-inducible lentiviral vector (kindly provided by J.P. Medema). The synthetic gene encoding CD44v6 (Eurofins MWG Operon) and PI3KCA cDNA (Addgene, 12523) were inserted into the pTWEEN EGFP expression vector.

shp63 (GTCATTTGATTCGAGTAGA) and scramble sequences were cloned in a lentiviral Lentilox 3.7 vector. shCD44v6 (ACAGATGGCATGAGGGATATC), 
shPI3K (GCGAAATTCTCACACTATTAT) and scramble sequences were cloned downstream U6 promoter of the lentiviral pLK0.1 vector.

Lentiviral supernatants were collected 48 hours after transfection of the packaging cell line HEK-293T. Transfection was performed by using X-treme GENE HP DNA Transfection Reagent (Roche). Cells $\left(1 \times 10^{5}\right)$ were then transduced with $1 \mathrm{ml}$ of viral supernatant for 24-48 hours using $8 \mu \mathrm{g} / \mathrm{mL}$ polybrene. All the experiments using transduced cells, started 5 days after the transduction. For the double transduction of inducible (iTAp63 and and $\mathrm{i} \Delta \mathrm{Np} 63$ ) and over-espressing/down-regulating CD44v6 (v6 and shv6) vectors, BCSCs were firstly transduced with iEV, iTAP63 and i $\triangle \mathrm{Np} 63$, and after one week with vectors coding for CD44v6 (v6) or shCD44v6 (shv6) and their controls (EV and Scr). After 7 days, doxycycline was added (to induce expression of the p63 isoforms) for 5 days.

\section{Cytokines quantification and cell treatment}

CAFs conditioned medium was derived from cells plated at approximately $70-80 \%$ of confluence in T25 flasks and grown for 48-hours in $10 \mathrm{ml}$ serum-free stem cell medium with $50 \mathrm{mg} / \mathrm{mL}$ ascorbic acid. Quantification of HGF, SDF-1 and OPN production of BCSCs or CAFs was measured using multiplex Bio-Plex Pro Assays (BioRad). Raw data (mean fluorescence intensity) from all kits were analyzed by Bio-Plex Software (Bio-Rad).

For cytokines treatment, BCSCs were starved of EGF and b-FGF overnight and then treated for 24 hours with HGF (100 ng/ml), SDF-1 (100ng/ml), OPN (1 $\mu \mathrm{g} /$ $\mathrm{ml}$ ), or CAF-CM. Treated cells were collected and used for RNA extraction.

\section{Animals and tumor models}

Mice experiments were performed according to the animal care committee guidelines of the University of Palermo. All the in vivo experiments were performed in 6 mice per condition using the 2 more tumorigenic luminal (patient \#4 and \#18, see Table 1) and 2 basal (patient \#10 and \#30) BCSCs. For the tumorigenic assay cells were harvested, suspended in $100 \mu \mathrm{l}$ of 1:1 Matrigel (BD Matrigel Matrix Growth Factor Reduced) and then injected subcutaneously into 5-week-old NOD/SCID mice (Charles River Laboratories, Milan, Italy). To test the invasive capacity of luminal BCSCs over-expressing $\triangle \mathrm{Np} 63$, a 1:1 mixture of EV-GFP:EV-RFP or TAp63-GFP: $\triangle N p 63-$ RFP transduced cells was suspended in Matrigel and subcutaneously injected in NOD/SCID mice. Tumor growth was followed over time after cell injection and their size was measured weekly using an electronic caliper. The volume was then calculated with the formula: largest measured diameter $\times(\text { smallest measured diameter })^{2} \times \pi / 6$.
Serial passages of tumors were performed by injecting 4,000 BCSCs subcutaneously in $100 \mu$ of 1:1 Matrigel. Sub-renal capsule injections were performed using 4,000 cells transduced with LUC/GFP in $50 \mu$ of 1:3 Matrigel. Tumor and metastasis formation was monitored over time and tracked in vivo by using the Photon IMAGER (Biospace Lab) for up to 14 weeks. At the endpoints, mice were sacrificed following IACUC guidelines, and xenograft tumor samples collected. Xenograft tumor tissues were collected and used for paraffin-embedded tissues, RNA, protein, and cell isolation. The survival rate was evaluated on the basis of photon emission, in particular the mice were sacrificed when the total amount of photons/s/sr of all secondary lesions was $4 \times 10^{3}$, which corresponded to $a \leq 0.5 \mathrm{~cm}^{2}$ tumor metastatic area.

\section{Statistical analysis}

Results are shown as the mean \pm SD for at least three repeated independent experiments for each group. The mean and SD were obtained by analyzing replicates using Prism 5 (GraphPad Software, La Jolla, CA, USA) applying two-tails Student's $t$-test for independent experiments. Significance levels were indicated as $P$ values. * indicates $P<0.05$, ** indicate $P<0.01$ and *** indicate $P<0.001$. ns indicates non statistically significant.

\section{ACKNOWLEDGMENTS}

We would like to thank Tatiana Terranova for manuscript editing and proofreading. We thank Francesco Calò for figures editing, and Elisa Lipari, Giovanni Tomaselli and Alessandro Gorgone for technical assistance.

\section{CONFLICTS OF INTEREST}

There is no conflicts of interest.

\section{FINANCIAL SUPPORT}

This work was supported by Associazione Italiana per la Ricerca sul Cancro (AIRC) to V.D.L. (AIRC IG 15196), G.S. (AIRC IG 16746 and MoH RF-201102349126) and M.T. (AIRC IG 14415), and by a grant of the National Fund against Cancer (SNFK) and Dutch Cancer Society Grants (UvA2009) 4416 and UvA20125425) to J.P.M. S.D.F. and A.T. are AIRC fellowship recipients.

\section{Author Contributions}

S.D.F., A.T.: conception and design, collection and/or assembly of data, data analysis, interpretation, 
manuscript writing; A.B., M.L.C.: collection and/or assembly of data, data analysis and interpretation; M.G., L.R.M., T.A., R.K. and A.C.: collection and/or assembly of data; D.B. provision of study material or patient; F.D.: financial support, administrative support; G.P., S.V. and E.G.: provision of study materials or patients; J.P.M.: financial support, administrative support, provision of study materials or patients; G.S.: conception and design, provision of study materials or patients; V.D.L. and M.T.: conception and design, financial support, administrative support, provision of study materials or patients, manuscript writing, final approval of manuscript.

\section{REFERENCES}

1. Fuchs E and Raghavan S. Getting under the skin of epidermal morphogenesis. Nature reviews Genetics. 2002; 3:199-209.

2. Mills AA, Zheng B, Wang XJ, Vogel H, Roop DR and Bradley A. p63 is a p53 homologue required for limb and epidermal morphogenesis. Nature. 1999; 398:708-713.

3. Yang A, Schweitzer R, Sun D, Kaghad M, Walker N, Bronson RT, Tabin C, Sharpe A, Caput D, Crum C and McKeon F. p63 is essential for regenerative proliferation in limb, craniofacial and epithelial development. Nature. 1999; 398:714-718.

4. Yang A, Kaghad M, Wang Y, Gillett E, Fleming MD, Dotsch V, Andrews NC, Caput D and McKeon F. p63, a p53 homolog at 3q27-29, encodes multiple products with transactivating, death-inducing, and dominant-negative activities. Molecular cell. 1998; 2:305-316.

5. Wu G, Nomoto S, Hoque MO, Dracheva T, Osada M, Lee CC, Dong SM, Guo Z, Benoit N, Cohen Y, Rechthand P, Califano J, Moon CS, Ratovitski E, Jen J, Sidransky D, et al. DeltaNp63alpha and TAp63alpha regulate transcription of genes with distinct biological functions in cancer and development. Cancer research. 2003; 63:2351-2357.

6. Melino G, Lu X, Gasco M, Crook T and Knight RA. Functional regulation of p73 and p63: development and cancer. Trends in biochemical sciences. 2003; 28:663-670.

7. De Laurenzi V and Melino G. Evolution of functions within the p53/p63/p73 family. Annals of the New York Academy of Sciences. 2000; 926:90-100.

8. De Laurenzi V, Rossi A, Terrinoni A, Barcaroli D, Levrero M, Costanzo A, Knight RA, Guerrieri P and Melino G. p63 and p73 transactivate differentiation gene promoters in human keratinocytes. Biochemical and biophysical research communications. 2000; 273:342-346.

9. Candi E, Rufini A, Terrinoni A, Dinsdale D, Ranalli M, Paradisi A, De Laurenzi V, Spagnoli LG, Catani MV, Ramadan S, Knight RA and Melino G. Differential roles of p63 isoforms in epidermal development: selective genetic complementation in p63 null mice. Cell death and differentiation. 2006; 13:1037-1047.
10. Nylander K, Vojtesek B, Nenutil R, Lindgren B, Roos G, Zhanxiang W, Sjostrom B, Dahlqvist A and Coates PJ. Differential expression of p63 isoforms in normal tissues and neoplastic cells. The Journal of pathology. 2002; 198:417-427.

11. Di Como CJ, Urist MJ, Babayan I, Drobnjak M, Hedvat CV, Teruya-Feldstein J, Pohar K, Hoos A and CordonCardo C. p63 expression profiles in human normal and tumor tissues. Clinical cancer research. 2002; 8:494-501.

12. Patturajan $M$, Nomoto $S$, Sommer M, Fomenkov A, Hibi K, Zangen R, Poliak N, Califano J, Trink B, Ratovitski E and Sidransky D. DeltaNp63 induces beta-catenin nuclear accumulation and signaling. Cancer cell. 2002; 1:369-379.

13. Rocco JW, Leong CO, Kuperwasser N, DeYoung MP and Ellisen LW. p63 mediates survival in squamous cell carcinoma by suppression of p73-dependent apoptosis. Cancer cell. 2006; 9:45-56.

14. Gressner O, Schilling T, Lorenz K, Schulze Schleithoff E, Koch A, Schulze-Bergkamen H, Lena AM, Candi E, Terrinoni A, Catani MV, Oren M, Melino G, Krammer $\mathrm{PH}$, Stremmel W and Muller M. TAp63alpha induces apoptosis by activating signaling via death receptors and mitochondria. The EMBO journal. 2005; 24:2458-2471.

15. Gallegos JR, Litersky J, Lee H, Sun Y, Nakayama $\mathrm{K}$, Nakayama $\mathrm{K}$ and $\mathrm{Lu} \mathrm{H}$. SCF TrCP1 activates and ubiquitylates TAp63gamma. The Journal of biological chemistry. 2008; 283:66-75.

16. Valent P, Bonnet D, De Maria R, Lapidot T, Copland M, Melo JV, Chomienne C, Ishikawa F, Schuringa JJ, Stassi G, Huntly B, Herrmann H, Soulier J, Roesch A, Schuurhuis GJ, Wohrer S, et al. Cancer stem cell definitions and terminology: the devil is in the details. Nature reviews Cancer. 2012; 12:767-775.

17. Zeuner A, Todaro M, Stassi G and De Maria R. Colorectal cancer stem cells: from the crypt to the clinic. Cell stem cell. 2014; 15:692-705.

18. Al-Hajj M, Wicha MS, Benito-Hernandez A, Morrison SJ and Clarke MF. Prospective identification of tumorigenic breast cancer cells. Proceedings of the National Academy of Sciences of the United States of America. 2003; 100:39833988.

19. Alison MR, Islam S and Wright NA. Stem cells in cancer: instigators and propagators? Journal of cell science. 2010; 123:2357-2368.

20. Chaffer CL, Brueckmann I, Scheel C, Kaestli AJ, Wiggins PA, Rodrigues LO, Brooks M, Reinhardt F, Su Y, Polyak K, Arendt LM, Kuperwasser C, Bierie B and Weinberg RA. Normal and neoplastic nonstem cells can spontaneously convert to a stem-like state. Proceedings of the National Academy of Sciences of the United States of America. 2011; 108:7950-7955.

21. Liu S, Cong Y, Wang D, Sun Y, Deng L, Liu Y, MartinTrevino R, Shang L, McDermott SP, Landis MD, Hong S, Adams A, D'Angelo R, Ginestier C, Charafe-Jauffret 
E, Clouthier SG, et al. Breast cancer stem cells transition between epithelial and mesenchymal states reflective of their normal counterparts. Stem cell reports. 2014; 2:78-91.

22. Todaro M, Gaggianesi M, Catalano V, Benfante A, Iovino F, Biffoni M, Apuzzo T, Sperduti I, Volpe S, Cocorullo G, Gulotta G, Dieli F, De Maria R and Stassi G. CD44v6 is a marker of constitutive and reprogrammed cancer stem cells driving colon cancer metastasis. Cell stem cell. 2014; 14:342-356.

23. Polyak K and Metzger Filho O. SnapShot: breast cancer. Cancer cell. 2012; 22:562-562 e561.

24. Engelman JA. Targeting PI3K signalling in cancer: opportunities, challenges and limitations. Nature reviews Cancer. 2009; 9:550-562.

25. Abd El-Rehim DM, Pinder SE, Paish CE, Bell J, Blamey RW, Robertson JF, Nicholson RI and Ellis IO. Expression of luminal and basal cytokeratins in human breast carcinoma. The Journal of pathology. 2004; 203:661-671.

26. Perou CM, Sorlie T, Eisen MB, van de Rijn M, Jeffrey SS, Rees CA, Pollack JR, Ross DT, Johnsen H, Akslen LA, Fluge O, Pergamenschikov A, Williams C, Zhu SX, Lonning PE, Borresen-Dale AL, et al. Molecular portraits of human breast tumours. Nature. 2000; 406:747-752.

27. Ginestier C, Hur MH, Charafe-Jauffret E, Monville F, Dutcher J, Brown M, Jacquemier J, Viens P, Kleer CG, Liu S, Schott A, Hayes D, Birnbaum D, Wicha MS and Dontu G. ALDH1 is a marker of normal and malignant human mammary stem cells and a predictor of poor clinical outcome. Cell stem cell. 2007; 1:555-567.

28. Chakrabarti R, Wei Y, Hwang J, Hang X, Andres Blanco M, Choudhury A, Tiede B, Romano RA, DeCoste C, Mercatali L, Ibrahim T, Amadori D, Kannan N, Eaves CJ, Sinha S and Kang Y. DeltaNp63 promotes stem cell activity in mammary gland development and basal-like breast cancer by enhancing Fzd7 expression and Wnt signalling. Nature cell biology. 2014; 16:1004-1015, 1001-1013.

29. Memmi EM, Sanarico AG, Giacobbe A, Peschiaroli A, Frezza V, Cicalese A, Pisati F, Tosoni D, Zhou H, Tonon G, Antonov A, Melino G, Pelicci PG and Bernassola F. p63 Sustains self-renewal of mammary cancer stem cells through regulation of Sonic Hedgehog signaling. Proceedings of the National Academy of Sciences of the United States of America. 2015; 112:3499-3504.

30. Giacobbe A, Compagnone M, Bongiorno-Borbone L, Antonov A, Markert EK, Zhou JH, AnnicchiaricoPetruzzelli M, Melino G and Peschiaroli A. p63 controls cell migration and invasion by transcriptional regulation of MTSS1. Oncogene. 2016; 35:1602-1608.

31. Dhasarathy A, Kajita M and Wade PA. The transcription factor snail mediates epithelial to mesenchymal transitions by repression of estrogen receptor-alpha. Molecular endocrinology. 2007; 21:2907-2918.

32. Boldrup L, Coates PJ, Gu X and Nylander K. DeltaNp63 isoforms regulate CD44 and keratins 4, 6, 14 and 19 in squamous cell carcinoma of head and neck. The Journal of pathology. 2007; 213:384-391.

33. Mani SA, Guo W, Liao MJ, Eaton EN, Ayyanan A, Zhou AY, Brooks M, Reinhard F, Zhang CC, Shipitsin M, Campbell LL, Polyak K, Brisken C, Yang J and Weinberg RA. The epithelial-mesenchymal transition generates cells with properties of stem cells. Cell. 2008; 133:704-715.

34. Baccelli I, Schneeweiss A, Riethdorf S, Stenzinger A, Schillert A, Vogel V, Klein C, Saini M, Bauerle T, Wallwiener M, Holland-Letz T, Hofner T, Sprick M, Scharpff M, Marme F, Sinn HP, et al. Identification of a population of blood circulating tumor cells from breast cancer patients that initiates metastasis in a xenograft assay. Nature biotechnology. 2013; 31:539-544.

35. Yuan TL and Cantley LC. PI3K pathway alterations in cancer: variations on a theme. Oncogene. 2008; 27:54975510.

36. Mayer IA, Abramson VG, Isakoff SJ, Forero A, Balko JM, Kuba MG, Sanders ME, Yap JT, Van den Abbeele AD, Li Y, Cantley LC, Winer E and Arteaga CL. Stand up to cancer phase Ib study of pan-phosphoinositide-3-kinase inhibitor buparlisib with letrozole in estrogen receptorpositive/human epidermal growth factor receptor 2-negative metastatic breast cancer. Journal of clinical oncology. 2014; 32:1202-1209.

37. Wallin JJ, Guan J, Prior WW, Lee LB, Berry L, Belmont LD, Koeppen H, Belvin M, Friedman LS and Sampath D. GDC-0941, a novel class I selective PI3K inhibitor, enhances the efficacy of docetaxel in human breast cancer models by increasing cell death in vitro and in vivo. Clinical cancer research. 2012; 18:3901-3911.

38. Guo X, Keyes WM, Papazoglu C, Zuber J, Li W, Lowe SW, Vogel H and Mills AA. TAp63 induces senescence and suppresses tumorigenesis in vivo. Nature cell biology. 2009; 11:1451-1457.

39. Charafe-Jauffret E, Ginestier C, Iovino F, Tarpin C, Diebel M, Esterni B, Houvenaeghel G, Extra JM, Bertucci F, Jacquemier J, Xerri L, Dontu G, Stassi G, Xiao Y, Barsky $\mathrm{SH}$, Birnbaum D, et al. Aldehyde dehydrogenase 1-positive cancer stem cells mediate metastasis and poor clinical outcome in inflammatory breast cancer. Clinical cancer research. 2010; 16:45-55.

40. Charafe-Jauffret E, Ginestier C, Iovino F, Wicinski J, Cervera N, Finetti P, Hur MH, Diebel ME, Monville F, Dutcher J, Brown M, Viens P, Xerri L, Bertucci F, Stassi $\mathrm{G}$, Dontu G, et al. Breast cancer cell lines contain functional cancer stem cells with metastatic capacity and a distinct molecular signature. Cancer research. 2009; 69:1302-1313.

41. Lodillinsky C, Infante E, Guichard A, Chaligne R, Fuhrmann L, Cyrta J, Irondelle M, Lagoutte E, Vacher S, Bonsang-Kitzis H, Glukhova M, Reyal F, Bieche I, Vincent-Salomon A and Chavrier P. p63/MT1-MMP axis is required for in situ to invasive transition in basal-like breast cancer. Oncogene. 2015. 
42. Du Z, Li J, Wang L, Bian C, Wang Q, Liao L, Dou X, Bian X and Zhao RC. Overexpression of DeltaNp63alpha induces a stem cell phenotype in MCF7 breast carcinoma cell line through the Notch pathway. Cancer science. 2010; 101:2417-2424.

43. Thiery JP, Acloque H, Huang RY and Nieto MA. Epithelialmesenchymal transitions in development and disease. Cell. 2009; 139:871-890.
44. Todaro M, Alea MP, Di Stefano AB, Cammareri P, Vermeulen L, Iovino F, Tripodo C, Russo A, Gulotta G, Medema JP and Stassi G. Colon cancer stem cells dictate tumor growth and resist cell death by production of interleukin-4. Cell stem cell. 2007; 1:389-402. 